\title{
Stress induced osmotic changes as a function of deprivation state*
}

\author{
EDWARD DEAUX, Antioch College, Yellow Springs, Ohio 45387 \\ and
}

JAN W. KAKOLEWSKI, Fels Research Institute, Yellow Springs, Ohio 45387

A series of experiments determined the relationship between the internal osmotic balance of rats and the direction and magnitude of stress-induced, body-fluid osmolality (BFO) changes. The state of BFO was varied by food or water deprivation. Two forms of stress were applied: rotation or sham stomach loading. The results indicate that animals deprived of food or water failed to respond to rotation, but deprivation conditions had no influence on the $\mathrm{BFO}$ change induced by sham stomach loading. Although the change of BFO in response to nonspecific, stressful stimuli appeared to be in the hyperosmotic direction, the magnitude of BFO increase was a function of the severity of stress and the state of BFO.

In two recent publications it was reported that stress increases the effective body-fluid osmolality (BFO) in rats. In the first (Deaux \& Kakolewski, 1970a), stress was provided by either handling the animals or rotating them in a container, and both behavioral and blood serum measures revealed an increased BFO following stress. In the second report (Kakolewski \& Deaux, $1970 \mathrm{~b}$ ), sham stomach loading was employed, and serum osmolality was found to be greatly elevated by the procedure. In both studies, however, serum measures of the BFO response to stress were determined only in animals maintained with free access to food and water.

The purpose of the present study was to establish if differences in the internal osmotic balance of the animal, produced by different feeding conditions, would influence the direction or magnitude of stress-induced osmotic changes. Groups of rats were water deprived, resulting in progressive dehydration (hyperosmolality), or food deprived, which resulted in gradual electrolyte loss (hypoosmolality). Control rats were kept ad lib and were, presumably, in an optimal osmotic balance, referred to as mesoosmolality (Deaux \& Kakolewski, 1970b), at the time of the experiment. Both enclosed rotation and sham stomach loading were employed as stressful situations, and serum osmolality was measured for all animals.

\section{SUBJECTS}

Seventy-six male Holtzman albino rats, 100-150 days of age, were used in the experiments. All animals were maintained in individual cages in a continuously lighted room kept at $72^{\circ} \mathrm{F}$.

*This research was supported in part by National Institute of Mental Health Grant M-4529. Research Grant NGL 36-005 from the $\mathrm{National}$ Aeronautics and Space Administration, and a College Science Improvement Program grant from the National Science Foundation.

\section{PROCEDURE}

The first experiment involved a 2 by 3 factorial design in which two conditions, stress and nonstress control, were combined with three internal states produced by food deprivation, water deprivation, or ad lib maintenance during the $24 \mathrm{~h}$ immediately preceding the experiment. Thirty-six rats were assigned randomly to the six groups with six animals per group. The control animals were sacrificed by decapitation as quickly as possible after being removed from their cages in order to provide blood for the determination of serum osmolality, which was made in an 0.4-ml sample of serum from each animal by means of an Advanced Instruments Osmometer. The animals in the stress condition were each placed in a weighing container from an $O^{\prime}$ Haus animal scale and rotated about several axes for $1.5 \mathrm{~min}$ before decapitation.

In the second experiment, 10 rats were assigned randomly to each of the four cells of a 2 by 2 factorial design, which combined the two conditions of stress and nonstress control with the two states of hunger and thirst, again provided by $24 \mathrm{~h}$ of food deprivation and water deprivation, respectively. Control animals were sacrificed by decapitation immediately after they were removed from their cages, and serum osmolality was determined as in the first experiment. Stress was provided in this experiment by sham stomach loading, conducted with a Goodyear 710-FS-10-AM elastic catheter, which preceded decapitation. The sham stomach-loading procedure lasted 20-60 sec. Since the identical technique had been used in a previous study (Kakolewski \& Deaux, 1970b) with mesoosmotic rats, it was unnecessary to include that aspect of the design in the current investigation. RESULTS

The group mean serum osmolalities from the first experiment are presented in Panel A of Fig. 1. It can be seen that among the three nonstress control groups the deprivation affected serum osmolality, with the food-deprived rats having an osmolality slightly lower than ad lib rats and water-deprived animals having an elevated serum osmolality. The effect of enclosed rotation was not found to be equal in the three stressed groups; no increment in serum osmolality occurred in food-deprived rats, and water-deprived rats responded with only a slight rise in osmolality relative to that found in the rats kept ad lib. An analysis of variance conducted on these data revealed a significant effect of rotation $(\mathrm{F}=4.23, \quad \mathrm{~d} f=1 / 30, \quad \mathrm{p}<.05)$, deprivation state $(\mathrm{F}=41.41$, $\mathrm{df}=2 / 30, \quad \mathrm{p}<.001)$, and the interaction of the two main effects $(F=3.43, \mathrm{df}=2 / 30, p<.05)$. Specific comparisons further indicated that the linear component of the deprivation-state effect accounted for $99.78 \%$ of the between-state variance, whereas the quadratic component of the interaction accounted for $96.48 \%$ of the Group by State variance. These verify the observable trends in the graphical representation of the results.

The data from the second experiment were combined with the mean serum osmolalities of the sham stomach-loaded and nonstress control groups from the earlier study by Kakolewski \& Deaux (1970b), and the resulting six group means are presented in Panel B of Fig. 1. Unlike the effect of enclosed rotation, sham stomach loading resulted in a large increment in serum osmolality in both deprivation states as well as in animals kept ad lib. An analysis of variance conducted on the 2 by 2 factorial (i.e., not including the data from the earlier report) yielded an $F$ of 119.24 $(\mathrm{df}=1 / 36, \quad \mathrm{p}<.001)$ for sham stomach loading and an $F$ of 8.74 $(\mathrm{df}=1 / 36, \quad \mathrm{p}<.01)$ for the deprivation state. The interaction of the two main effects was not significant $(F<1.0)$, verifying the equal increments which can be seen in the figure.

The results indicate that the effect of stress was an increase in body-fluid osmolality (BFO) but that the effect did not occur consistently. The increase following enclosed rotation was largely dependent on an animal's state of osmotic balance, whereas the rise in osmolality after sham stomach loading was not state dependent. A reasonable explanation for this difference would attribute it to the difference in the severity of stress; $\mathrm{BFO}$ is increased by a relatively mild level of stress only when, at the onset of stress, an animal is mesoosmotic, at which time it could be said it is neither hungry nor thirsty (Deaux \& 


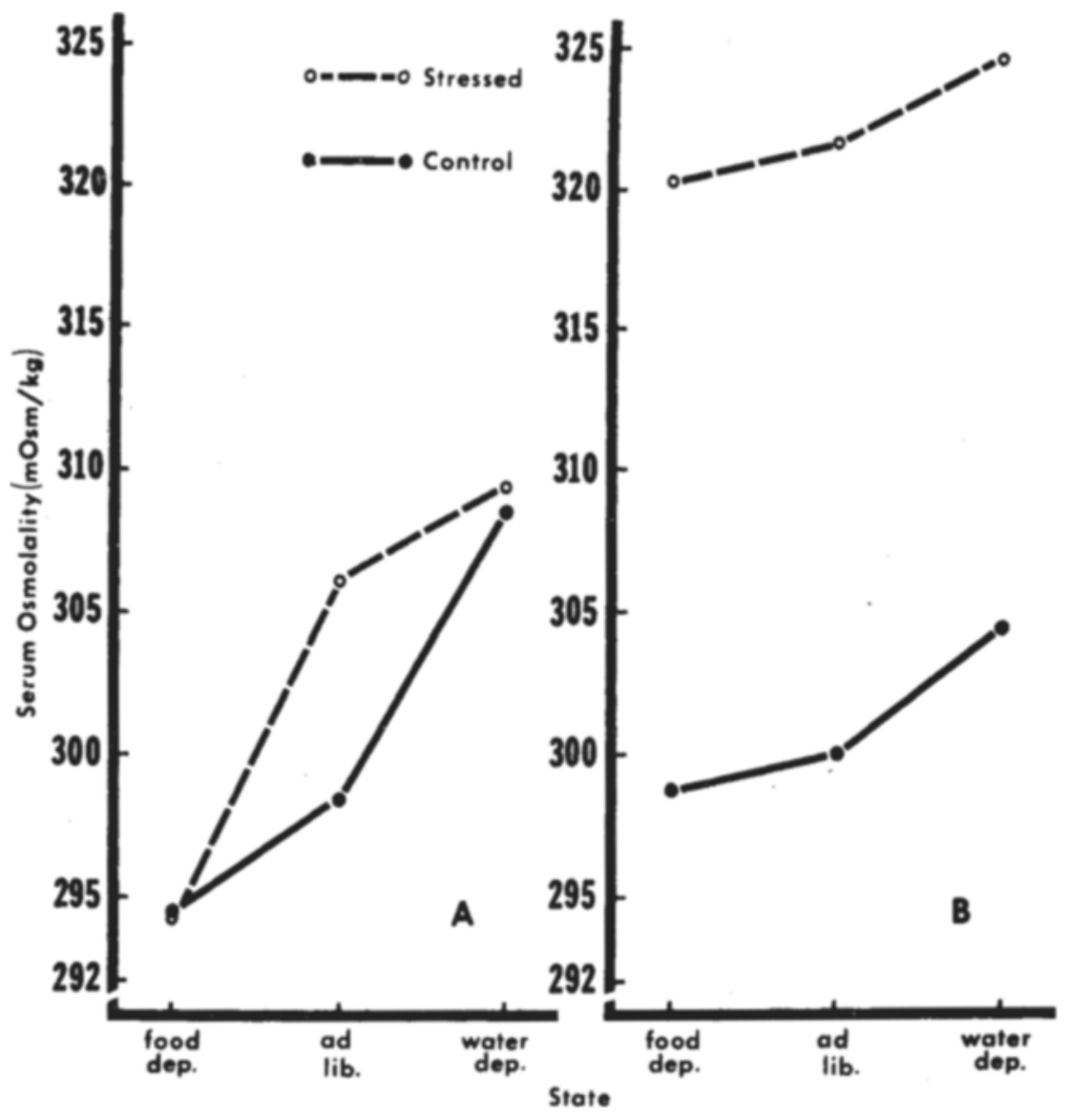

Fig. 1. Panel A: Group-mean serum osmolalities for the six groups of Experiment 1, in which stress was provided by enclosed rotation. Panel B: Mean serum osmolalities of the four groups of Experiment 2 plus the two ad lib groups from Kakolewski \& Deaux (1970b). Stress was provided by sham stomach loading.

Kakolewski, 1970b). On the other hand, a relatively severe stress triggers an increase in BFO, regardless of an animal's osmotic state. That the severity of stress was greater during the sham stomach-loading procedure than during enclosed rotation is substantiated by several behavioral indices (e.g., squealing, biting, urinating, and defecation). Likewise, with the ad lib animals, the mean increase in serum osmolality was 2.7 times greater following sham stomach loading than it was following enclosed rotation. Thus, both behavioral and physiological responses can be viewed as supporting this interpretation.

At present, the incremental effect of stress on BFO must still be attributed to nonspecific stimuli which occur in the process of the stressful manipulations employed. It can be suggested that the attenuating influence of osmotic imbalance (i.e., the states of hypo- and hyperosmolality) may be a function of a system which restricts stimulus input or in which sensitivity to. stimuli decreases when osmotic imbalance obtains. Only severely stressful situations provide stimuli of sufficient intensity or quantity to result in an increased BFO. However, it has been shown that stimuli specific to the need state of an animal have a rapid effect on BFO in the states of both hypoosmolality (Kakolewski \& Deaux, 1970a) and hyperosmolality (Deaux, Kakolewski, \& Sato, 1970), which supports the rather obvious fact that BFO is most responsive to specific osmotic input provided by food and water, respectively. Further work is in progress to attempt to delineate the stimuli which are necessary and sufficient to trigger this internal response.

A question which must be considered with regard to this phenomenon is its adaptiveness in the Darwinian sense. It seems unreasonable that selection pressure would encourage the interruption of either fight or flight to satisfy the induced state of thirst provided by a stressful encounter. Nevertheless, our earlier experiments have demonstrated that stress-induced thirst does occur (Deaux \& Kakolewski, 1970a). Viewed as a primary response, which by its effect triggers other secondary responses, its value in the behavioral repertoire of an animal may be appreciated.

Since Verney's studies (1947), it has been widely accepted that "painful" stimuli induce water retention due to a release of antidiuretic hormone $(\mathrm{ADH})$, which is also released following hyperosmolality provided by nonstress procedures (e.g., water deprivation). Further, the stress associated with sham stomach loading was observed to produce antidiuresis in a study by Kakolewski \& Valenstein (1969), who did not find antidiuresis in response to brief handling in overhydrated (hypoosmotic) rats. The latter results correspond well with the failure to find BFO increase in the food-deprived rotated animals in the present experiment. It would appear, then, that the release of $A D H$ in response to nociceptive stimuli may be due to the $\mathrm{BFO}$ increase rather than to a direct activation of the ADH system. It is also possible that other responses of an adaptive nature likewise are triggered by the rapid osmotic increase, in which case the importance of this phenomenon as a mediating response would be assured.

\section{REFERENCES}

DEAUX, E., \& KAKOLEWSKI, J. W. Emotionally induced increases in effective osmotic pressure and subsequent thirst. Science, 1970a, 169,1226-1228.

DEAUX, E., \& KAKOLEWSKI, J. W. The character of osmotic changes resulting in the initiation of eating. Journal of Comparative \& Physiological Psychology, $1970 \mathrm{~b}$, in press.

DEAUX, E., KAKOLEWSKI, J. W., \& SATO, E. Emergence of systemic cues evoking food-associated drinking. Physiology \& Behavior, 1970, 5, 1177-1179.

KAKOLEWSKI, J. W., \& DEAUX, E. Initiation of eating as a function of ingestion of hypoosmotic solutions. American Journal of Physiology, 1970a, 218,590-595.

KAKOLEWSKI, J. W., \& DEAUX, E. Influence of sham stomach-loading on serum osmolality. Physiology \& Behavior, 1970b, 5, 1205-1206.

KAKOLEWSKI, J. W., \& VALENSTEIN, E. $S$. Antidiuresis associated with the ingestion of food substances. In C. Pfaffman (Ed.), Olfaction and taste. Rock efeller University Press, 1969. Pp. 593-600.

VERNEY, E. B. The antidiuretic hormone and the factors which determine its release. Proceedings of the Royal Society (London), 1947, $135 \mathrm{~B}, 25-106$. 